\title{
Synthetic parasites: a successful mucosal nanoparticle vaccine against Toxoplasma congenital infection in mice
}

\author{
Céline Ducournau ${ }^{* \neq, 1,}$, Thi TL Nguyen ${ }^{\ddagger 1}$, Rodolphe Carpentier ${ }^{2,5}$, \\ Isabelle Lantier ${ }^{3}$, Stéphanie Germon', Flavien Précausta', Pierre-Jean Pisella', \\ Hervé Leroux ${ }^{3}$, Nathalie Van Langendonck ${ }^{4}$, Didier Betbeder ${ }^{2,5}$ \\ \& Isabelle Dimier-Poisson'
}

\begin{abstract}
Aim: Development of protein vaccine to prevent congenital infection is a major public health priority. Our goal is the design of mucosal synthetic pathogen inducing protective immune responses against congenital toxoplasmosis. Materials \& methods: Mice were immunized intranasally, establishing pregnancy and challenging orally. Placental immune response, congenital infection, pup growth, parasitic load rates were studied. Results: Pups born to vaccinated infected dams had significantly fewer brain cysts, no intraocular inflammation and normal growth. Protection was associated with a placental cellular Th1 response downregulated by IL- 6 and correlated with persistence of vaccine for few hours in the nose before being totally eliminated. Conclusion: Our vaccine conferred high protection against congenital toxoplasmosis. These results provide support for future studies of other congenital vaccine.
\end{abstract}

First draft submitted: 21 July 2016; Accepted for publication: 17 November 2016; Published online: 24 March 2017

\section{Background}

There is pressing need to develop effective vaccines against intracellular pathogens [1]. Moreover, congenital infectious diseases (malaria, trypanosomiasis, cytomegalovirus infection, pertussis, toxoplasmosis...) are a leading worldwide cause of morbidity and mortality in neonates [2]. Development of a vaccine focused on protecting fetuses and newborns from the sequel of congenital infections is, therefore, a major public health priority as identified by the National Vaccine Program Office and the Institute of Medicine [3]. Among congenital infections, toxoplasmosis due to the intracellular protozoan Toxoplasma gondii is considered as one of the five neglected parasitic infections targeted by Center of Disease Control (CDC) for public health action [4]. Indeed, T. gondii infection during pregnancy can result in spontaneous abortion, fetal or neonatal death and severe defects, such as hydrocephalus, chorioretinitis, blindness and mental retardation in newborns $[5,6]$. Congenital toxoplasmosis is also a serious cause of abortion and neonatal mortality

'Immunologie Parasitaire et Vaccinologie, Biothérapies Anti-Infectieuses, Université de Tours-INRA, UMR1282 Infectiologie et Santé Publique, UFR Pharmacie, F-37000 Tours, France

${ }^{2}$ Centre International de Recherche sur I'Inflammation de Lille LIRIC -UMR 995 Inserm / Université Lille 2 / CHRU Lille. Innovation thérapeutique ciblant l'inflammation. Groupe Nanomédecine, Faculté de Médecine, F-59045 Lille Cedex, France

${ }^{3}$ Laboratoire d'Expertise en Infection Animale, INRA-Université de Tours, UMR1282 Infectiologie et Santé Publique, F-37380 Nouzilly,

France

${ }^{4}$ Service de Parasitologie-Mycologie-Médecine Tropicale, CHRU, Tours, France

5Université d'Artois, rue du Temple, 62030 ARRAS, France

*Author for correspondence: Tel.: 33247367 277; celine.ducournau@univ-tours.fr

${ }^{\ddagger}$ Authors contributed equally

\section{KEYWORDS}

- congenital toxoplasmosis

- nanoparticles • nasal

vaccination 
in sheep and goats $[7,8]$. If there is evidence that maternal treatment reduces partially the risk of fetal infection due to an early diagnosis, vaccination remains the safest way of protecting fetuses against Toxoplasma infection $[9,10]$. However, as primary $T$. gondii infection leads to an immune response that confers lifelong protection against reinfection and congenital transmission, it should be possible to develop effective vaccine against this disease. A strain known as Mic1.3KO - derived from the highly virulent type I RH strain - was obtained in our laboratory by deleting the MIC1 and MIC3 genes [11]. This live vaccine protects totally pregnant sheep against toxoplasmosis and represents an attractive veterinary vaccine [12]. However, in human medicine, people who have damaged or weakened immune systems cannot safely receive this live vaccine. For this reason, we investigated research toward a protein vaccine which is extremely safe and devoid of side effects.

In recent years, nanoparticles have been increasingly studied for use in vaccine application $[13,14]$. Moreover, mucosal immunization using nanoparticles is of special interest to improve delivery of subunit vaccine through the epithelium, to enhance antigen immunogenicity and to offer the possibility of needle-free vaccinations [15]. Recently, we developed a parasitemimicking nanoparticle-based vaccine platform against toxoplasmosis [16]. Our platform consists of maltodextrin/phospholipid nanoparticles able to deliver all parasite antigens within mucosa and to induce specific humoral and Th1/Th17 cellular immune responses. Vaccination led to $100 \%$ survival of mice with acute toxoplasmosis and significant reduction in the number of brain cysts of mice with chronic toxoplasmosis [16]. Due to a lack of knowledge on maternal immune responses required for avoiding congenital transmission of $T$. gondii, protection against acute and chronic toxoplasmosis does not predict vaccine efficiency against congenital toxoplasmosis. The aim of this work was to evaluate the efficacy of our vaccine platform to protect fetuses against congenital toxoplasmosis. Moreover, the biodistribution of nanoparticles after their administration was evaluated and the safety issue was discussed.

\section{Methods}

\section{- Mice}

Eight-week-old male and female inbred CBA/J mice and female outbred Swiss mice were purchased from CER Janvier (Le Genest Saint Isle, France) and maintained under pathogenfree conditions in the animal house of the University of Tours. Experiments were carried out in accordance with the guideline for animal experimentation (EU Directive 2010/63/ EU) and the protocol was approved by the local ethics committee (CEEA VdL). As efficiency of nanoparticle vaccine was evaluated in acute and chronic forms of toxoplasmosis with CBA/J mice, these mice were also used in congenital toxoplasmosis [16]. Moreover, they represent a pertinent model because of the low rate of placental parasite transmission as in humans and CBA/J pups born from infected dams have a detectable parasite brain load allowing evaluating the vaccine efficacy. Concerning Di-palmitoyl phosphatidyl glycerol-loaded nanoparticles (DGNP) labeling and in vivo biodistribution study Swiss mice were used because of a better spectral absorbance due to their white pigmentation.

\section{- DGNP labeling \& in vivo biodistribution study in mice}

DGNPs made of a reticulated maltodextrin matrix with a lipidic core, were prepared as previously described [17]. DiD ( $\lambda$ ex: $648 \mathrm{~nm}, \lambda$ ex: $665 \mathrm{~nm}$, stock solution at $1 \mathrm{mg} / \mathrm{ml}$ in ethanol) was prepared at $35 \mu \mathrm{g} / \mathrm{ml}$ free in phosphatebuffered saline or incorporated into DGNP at $5 \mathrm{mg} / \mathrm{ml}$ (DiD loading was confirmed by gel filtration analysis using PD10 Sephadex columns, data not shown). The fluorescence was analyzed on a Fluoroskan ${ }^{\circledR}$ Ascent (ThermoFisher Scientific) in $100 \mu \mathrm{l}$ of each preparation.

Nonanesthetized Swiss mice were administered with $20 \mu \mathrm{l}$ of DGNP/DiD by nasal instillation, while negative control mice received $20 \mu \mathrm{l}$ of free DiD. Mice were imaged at 15 and $30 \mathrm{~min}$, $1,2,4,6,24,48$ and $72 \mathrm{~h}$ following instillation using an intensified charged coupled device camera of the in vivo Imaging System IVIS ${ }^{\circledR}$ Spectrum (PerkinElmer, MA, USA). Data were analyzed using Living Image ${ }^{\circledR} 4.3 .1$ software, and expressed as fluorescent units. The DGNP/ DiD were represented in a color scale from violet (low fluorescence intensity) to yellow (strong fluorescence intensity).

Mice were sacrificed after 2, 4, 6 and $24 \mathrm{~h}$, and nasal cavities, salivary glands, lung, spleen, liver, stomach, intestines and mesenteric lymph nodes were imaged ex vivo under the same conditions as the anesthetized animals. 


\section{- Preparation of vaccine formulation}

Total antigen extract (TE) of $T$. gondii was obtained as previously described from RH strain (type 1) grown in human foreskin fibroblasts [18]. DGNP/TE association was obtained by simple mixing of DGNP with TE as already described [16]. This association is due to electrostatic interactions of antigens with the cationic polysaccharide core of DGNP and/or with anionic DPPG, but hydrophobic interactions with phospholipids may also occur. A formulation of DGNP/TE 5:1 (w/w) was made by mixing $50 \mu \mathrm{g}$ of DGNP with $10 \mu \mathrm{g}$ of ice-cold TE. Prior to formulation, TE antigens were disaggregated by the addition of Empigen BB detergent $(0.5 \% \mathrm{v} / \mathrm{v})$ followed by three rounds of 1 -min sonication.

\section{- Immunization}

Females CBA/J mice $(\mathrm{n}=15)$ received at 2 week intervals two intranasal doses of $50 \mu \mathrm{g}$ DGNP, $10 \mu \mathrm{g}$ TE or the DGNP/TE formulation $(50 \mu \mathrm{g}$ DGNP/10 $\mu \mathrm{g}$ TE). Each mouse received $6 \mu \mathrm{l}$ per nostril. Nonimmunized mice served as controls: noninfected pregnant (NIP) or infected pregnant (IP). Three independent experiments have been done.

\section{- Mating \& challenge}

Two months after the second immunization, the female CBA/J mice were allowed to mate for 7 days following the synchronization of estrus cycle. Day 1 of the gestation (G1) was established by the detection of a mucous plug. The mice were orally challenged at mid-gestation (G11) with 15 cysts of a type 2 strain of $T$. gondii (76K strain maintained in CBA/J mice [19]). This low dose of infection was chosen to avoid abortion and to maintain gestation to term in order to observe the clinical development of illness in newborns.

\section{- Evaluation of protection against congenital toxoplasmosis}

Protection against congenital infection was evaluated at G17 on fetuses delivered by transabdominal incision of five pregnant females. Parasites were detected in fetal tissues by real-time PCR and local immune response was evaluated by cytokine secretion in placentas.

\section{- Parasite detection in fetuses by real-time PCR}

DNA extraction was realized with the kit Genomic DNA from Tissue (Macherey-Nagel,
Germany). Real time PCR was performed on $1 \mu \mathrm{g}$ of total DNA in a total volume of $25 \mu \mathrm{l}$ containing Platinium ${ }^{\circledR}$ Quantitative PCR Super Mix-UDG (Uracil-DNA glycosylase) (Invitrogen, USA), $4 \mathrm{mM} \mathrm{MgCl}, 0.5 \mu \mathrm{M}$ of each primer specific for the $18 \mathrm{~s} \mathrm{rDNA}$ gene of T. gondii TG III (5'-CCT TGG CCG ATA GGT CTA GG-3'); TG IIb (5'-GGC ATT CCT CGT TGA AGA TT-3'), and $180 \mathrm{nM}$ of the probe 5'-TGC AAT AAT CTA TCC CCA TCA CGA TGC ATA CTC AC-3' modified with 5'-FAM and 3'-TAMRA as reporter and quencher, respectively [20]. After initial UDG incubation for $2 \mathrm{~min}$ at $50^{\circ} \mathrm{C}$ and denaturation for $5 \mathrm{~min}$ at $95^{\circ} \mathrm{C}$, the two-step amplification conditions were 50 cycles of $20 \mathrm{~s}$ at $95^{\circ} \mathrm{C}$ and $60 \mathrm{~s}$ at $65^{\circ} \mathrm{C}$ with the Light $\mathrm{Cycler}^{\circledR} 480$ RealTime PCR System (Roche Applied Science, Germany). A standard curve for parasite number equivalence was generated in parallel.

\section{- Placental cytokine assay}

Half placental tissues were homogenized in $0.4 \mathrm{ml}$ of lysis buffer (Tris $50 \mathrm{mM}, \mathrm{NaCl} 150 \mathrm{mM}$, Nonidet P-40 1\%, pH 8) by using Gentle Macs (Miltenyi Biotech, Germany). After centrifugation at $13,000 \times \mathrm{g}$ for $10 \mathrm{~min}$, cell-free supernatants were harvested for determining levels of IFN- $\gamma$, IL-17, IL-10, IL-2, IL-4, IL-6, TNF- $\alpha$ and TGF- $\beta 1$ cytokines by using ELISA method.

\section{- Evaluation of protection against chronic toxoplasmosis}

Protection against chronic toxoplasmosis was evaluated after natural delivery in offspring and in dams. Offspring were weighed 2 weeks after birth. Offspring were sacrificed at the age of 8 weeks. The eyes were harvested and hydrated with sterile water. Inflammatory changes were observed in anterior and posterior segments by using a binocular magnifying lens. The pathological changes were scored on a scale from 0 to 4 [21]. Brain was homogenized in $5 \mathrm{ml}$ of Roswell Park Memorial Institute (RPMI) medium with a pestle and a mortar. The mean number of cysts per brain was determined by counting eight samples $(10 \mu \mathrm{l}$ each) of each homogenate under a microscope. Cysts were also counted in the brain of dams 2 months after infection.

\section{- Statistical analysis}

Kruskal-Wallis test was used to determine the significance of variations between groups with GraphPad Prism software. 


\section{Results}

- DGNP labeling \& in vivo biodistribution study in mice

DiD is a well-known fluorophore that emits fluorescence when it is associated to lipid membrane. In order to discriminate the fluorescence observed in vivo, free and DGNP-loaded DiD was analyzed. Free DiD showed a fluorescence intensity of 0.51 fluorescence unit (F.U.). When filtered, insoluble DiD was removed and fluorescence decreased close to background (0.27 F.U.). When DiD is loaded into DGNP, the fluorescence drastically increased (16.4 F.U. means $\times 32)$ and filtration did not evidence free DiD (15.9 F.U.). These results indicate that free DiD does not fluoresce in solution and that DiD fluorescence observed is restricted to DGNP loading (Figure 1).

In vivo experiments were carried out in order to follow the biodistribution of DGNP in animals. DGNP/DiD were administered nasally in nonanesthetized mice and the animals were imaged during $72 \mathrm{~h}$. As shown in Figure 2A, DGNP/DiD were present after $15 \mathrm{~min}$ in the nose where they remained for $6 \mathrm{~h}$, were thereafter found to undergo rapid transport into the gastrointestinal tract, and were totally eliminated via feces. As expected, no signal was observed when free DiD was administered as control.

As shown in Figure 2B, ex vivo imaging of organs autopsied at 2, 4, 6 and 24 h after administration confirmed in vivo experiments and showed a progressive elimination of DGNP/ $\mathrm{DiD}$ via the digestive tract; no other organs were labeled.

These results demonstrated the presence of DGNP/DiD in the nose after $6 \mathrm{~h}$, emphasizing the long residence time of the DGNP/DiD in the nasal cavity. On the other hand, $24 \mathrm{~h}$ after the administration, the DGNP/DiD presence was not revealed in tissues other than the digestive tract, suggesting that DGNP/DiD are rapidly eliminated gastrointestinally.

\section{- Impact of vaccination \&/or infection on fertility/prolificacy rates \& embryo resorption}

Pregnant mice immunized by the nasal route with DGNP, TE or DGNP/TE were orally infected with 15 cysts of $76 \mathrm{~K} T$. gondii strain on G11. The groups of NIP and IP served as nonimmunized controls. The effect of vaccination and/or infection with a low dose of cysts on pregnancy was studied by determining fertility

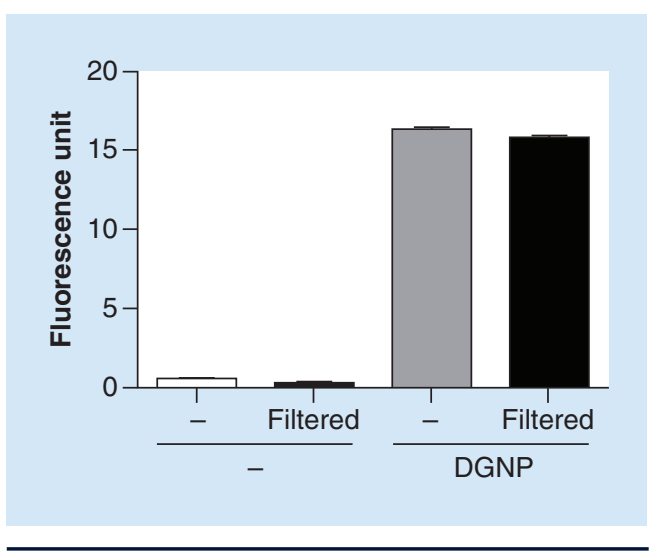

Figure 1. Fluorescence of free DiD or Dipalmitoyl phosphatidyl glycerol-loaded nanoparticles/DiD. Same doses of free DiD or DGNP/DiD were analyzed by spectrofluorimetry before and after $0.22 \mu \mathrm{m}$ filtration. Results are expressed as mean \pm SD.

DGNP: Di-palmitoyl phosphatidyl glycerolloaded nanoparticle.

and prolificacy rates as embryo resorption. The experimental results are shown in Table 1.

First, we observed that vaccination does not impair fertility in mice as the number of pregnant females was quite similar in each group of mice. The prolificacy of mice (mean number of offspring per litter) and the number of fetal resorptions per litter were also quite similar, whatever the group of mice examined. These results showed that vaccination and infection with a low dose of cysts had no impact on these parameters.

\section{- Evaluation of protection against congenital toxoplasmosis}

Regarding vertical transmission, the presence of $T$. gondii parasites was assessed in all the fetuses. As shown in Figure 3, parasite load was significantly lower in fetuses from DGNP/TE immunized mice $(37 \pm 11)$ compared with the other groups $(262 \pm 27$ for fetuses of IP group, $223 \pm 45$ for fetuses of DGNP group and $191 \pm 46$ for fetuses of TE group; $\mathrm{p}<0.0001$ Kruskal-Wallis), whereas only a slight decrease in parasite load is observed in fetuses of the TE group compared with fetuses from IP ( $p<0.05$ Kruskal-Wallis). Immunization with DGNP/TE therefore greatly reduced vertical transmission of T. gondii parasite.

\section{- Placental cellular immune response}

It is well known that the placenta plays a key role in the pathogenesis of congenital infections, 
(A)

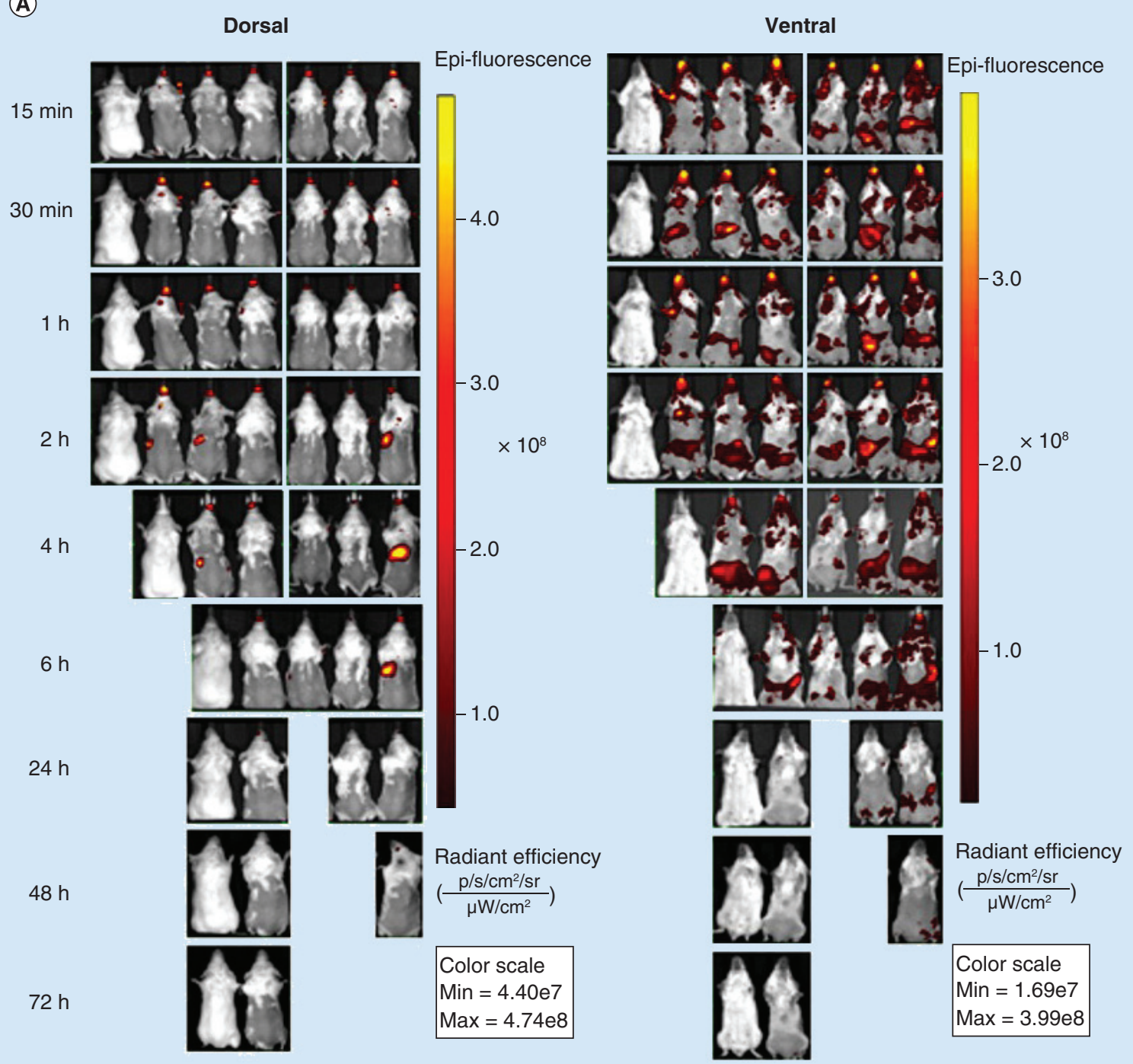

Figure 2. In vivo and ex vivo imaging of Di-palmitoyl phosphatidyl glycerol-loaded nanoparticles/DiD. (A) Mice were administered nasally with $12 \mu \mathrm{l}$ of DiD alone (control) or $12 \mu \mathrm{l}$ of DGNP/DiD and imaged using the IVIS Spectrum imager (PerkinElmer, MA USA). Fluorescence intensities of DGNP/DiD were represented from purple (low) to yellow (high). Whole body fluorescence imaging of the mice at 15 and $30 \mathrm{~min}, 1,2,4,6,24,48$ and $72 \mathrm{~h}$ following administration (dorsal left and ventral right). Ex vivo imaging of organs from the mouse autopsied at 2, 4, 6 and $24 \mathrm{~h}$ after instillation (B).

DGNP: Di-palmitoyl phosphatidyl glycerol-loaded nanoparticle.

however the factors controlling this process are not completely understood. To investigate the effect of vaccination on the placenta immune response, we measured the levels of placental selected cytokines (Th1 cytokines: IFN- $\gamma$ and TNF- $\alpha$, Th2 cytokine: IL-4, Treg cytokines: TGF- $\beta$ and IL-10, Th17 cytokine: IL-17A, and anti-inflammatory cytokine: IL-6) on G17. IL-4, TNF- $\alpha$ and IL-17A were not detectable in placental samples. No significant difference
( $\mathrm{p}=0.056$ Kruskal-Wallis) in production of placental TGF- $\beta$ was observed between the groups of mice (as shown in Figure 4A).

IFN- $\gamma$ was not found in placentas of NIP (Figure 4B). Specific and high production of IFN- $\gamma$, known to be harmful for pregnancy and to facilitate parasite transmission to the fetus [22], was observed in placentas of IP and DGNPimmunized dams. Very low levels of IFN- $\gamma$ were measured in placentas from TE and DGNP/TE 


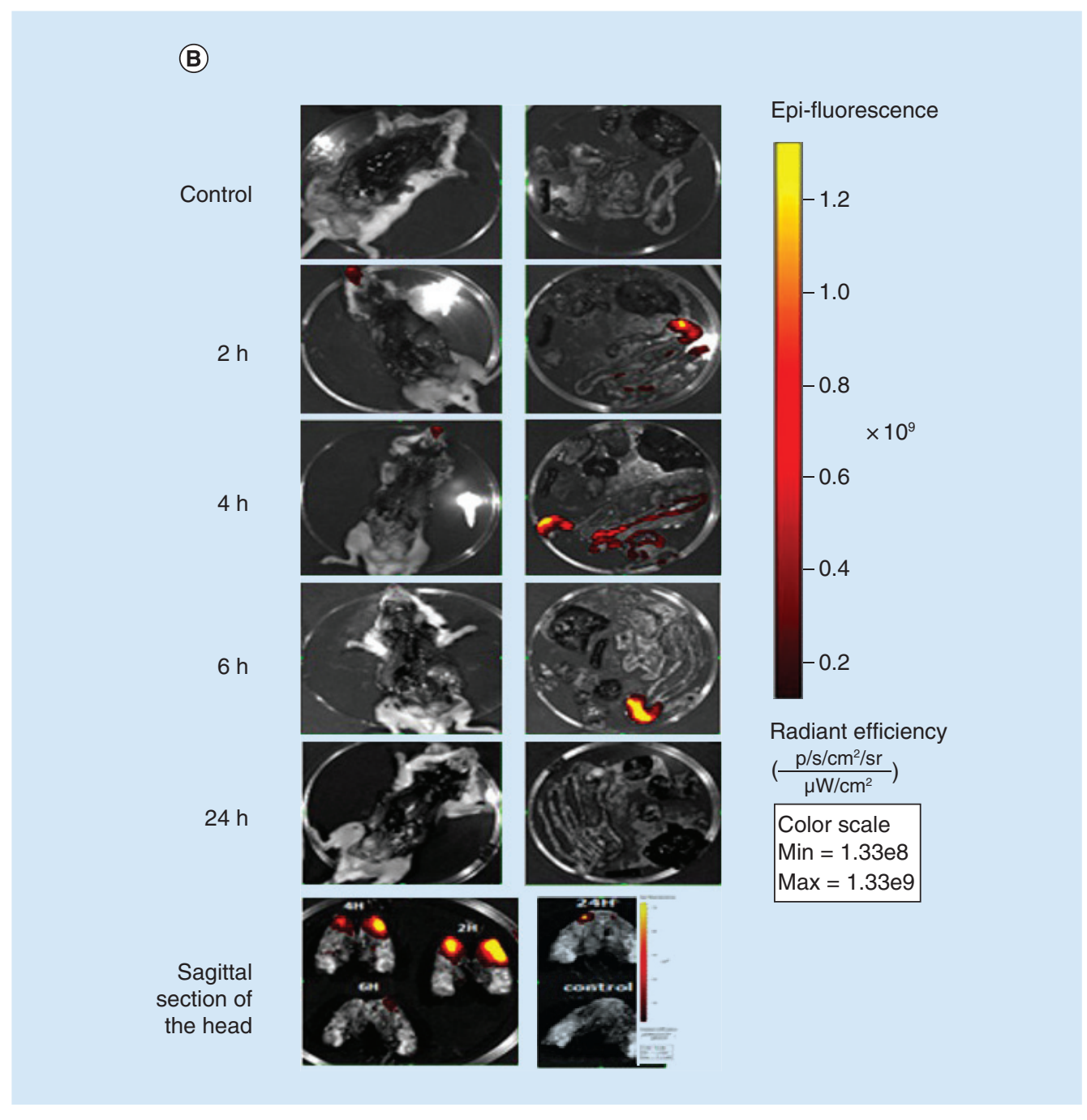

Figure 2. In vivo and ex vivo imaging of Di-palmitoyl phosphatidyl glycerol-loaded nanoparticles/ DiD (cont.). (B) Ex vivo imaging of organs from the mouse autopsied at 2, 4, 6 and $24 \mathrm{~h}$ after instillation.

DGNP: Di-palmitoyl phosphatidyl glycerol-loaded nanoparticle.

groups in comparison with the control groups ( $\mathrm{p}<0.0001$ Kruskal-Wallis).

Additionally, low levels of IL-6 (Figure 4C) were quantified in the placentas of noninfected mice and of all groups of infected mice except those immunized with DGNP/TE (p $=0.025$ Kruskal-Wallis). These results suggest that immunization with DGNP/TE induces IL- 6 that could play a critical role in the barrier defense against the parasite infection. In addition, placentas of DGNP/ TE-immunized mice secreted high levels of IL-10 but in the same extent than placentas of TE-immunized mice and NIP (Figure 4D: $\mathrm{p}<0.0001$ Kruskal-Wallis).
This shows that vaccination with DGNP/ TE both reduces proinflammatory and increases anti-inflammatory placental cytokine levels, facilitating the maintenance of gestation.

\section{- Evaluation of protection against chronic toxoplasmosis in offspring \& dams}

To evaluate vaccine efficacy in protecting neonates against abnormal growth due to $T$. gondii infection, we first calculated the average weight of each litter at 7,14 and 21 days. On the 14th day after birth, the mean weight of the offspring born from mice vaccinated with DGNP/TE was significantly higher $(6.58 \pm 0.17 \mathrm{~g})$ than those from mice of the IP, DGNP and TE groups 
Table 1. Impact of vaccination and/or infection on fertility/prolificacy rates and embryo resorption.

\begin{tabular}{llllll|} 
& IP & DGNP & TE & DGNP/TE & NIP \\
Number of litters/total number of females & 70 & 60 & 60 & 60 & 80 \\
\hline Mean of pups/litter & 4 & 3 & 3 & 3.3 & 4.7 \\
Fetal resorption (\%) & 22 & 27 & 39 & 16 & 29 \\
\hline $\begin{array}{l}\text { DGNP: Di-palmitoyl phosphatidyl glycerol-loaded nanoparticle; IP: Infected pregnant; NIP: Noninfected pregnant; TE: Total antigen } \\
\text { extract. }\end{array}$
\end{tabular}

$(4.78 \pm 0.12 \mathrm{~g}, 5.41 \pm 0.08 \mathrm{~g}$ and $5.45 \pm 0.09 \mathrm{~g}$, respectively; ${ }^{* * *} \mathrm{p}<0.0001$ Kruskal-Wallis) (Figure 5A). The weight loss came with clinical signs compatible with toxoplasmosis including rough hair coats, reluctance to move and signs of nerve damage (not shown). Noteworthy, there was no significant difference in weight of offspring from NIP mice $(6.62 \pm 0.3 \mathrm{~g})$ and from mice vaccinated with DGNP/TE.

One of the most common findings in congenital toxoplasmosis is retinochoroiditis, an ophthalmologic manifestation of the disease. To evaluate the protective effect of vaccination against ocular toxoplasmosis, determination of ophthalmologic clinical signs was performed under a binocular magnifying glass on each eye of each mouse 8 weeks after birth.

As shown in Figure 5C, inflammation in the eye tissues of mice from DGNP- and TE-immunized mice was significantly more severe than in those of infected mice vaccinated with DGNP/TE. In almost all cases, eyes from the DGNP/TE group did not develop ocular lesions (only $7 \%$ of ocular inflammation), whereas the percentage of lesions in the other groups was dramatically higher $(56 \%$ for TE, $42 \%$ for DGNP and $60 \%$ for IP groups) with concomitant secondary cataracts $(34,24$ and $37 \%$, respectively), severe vitreous and/or dilatation of iris vessels and/or conjunctiva-sclera (22, 18 or $23 \%$, respectively) (Figure 5D). Based on the mean score of ocular pathology, we observed an important decrease in ocular pathological score in the animals born to dams vaccinated with DGNP/TE (mean score $=0.28$ ) in comparison with those within the other groups (mean score $=1.8$ for TE and 1.3 for DGNP) (data not shown). Thus, vaccination with DGNP/TE protected against ocular toxoplasmosis resulting from congenital infection.

To further evaluate the capacity of the DGNP/TE vaccine to protect against chronic toxoplasmosis, parasite burden was studied in brain tissue of offspring and dams 2 months after delivery.
The mean number of cerebral cysts diminished significantly (corresponding to a 55\% decrease) in the offspring born to the dams vaccinated with DGNP/TE ( $60 \pm 8$ cysts per brain) in comparison with those from the other groups $(133 \pm 28$ for IP, $98 \pm 13$ for DGNP and $86 \pm 21$ for TE groups; ${ }^{*} \mathrm{p}=0.03$ Kruskal-Wallis) (Figure 6A).

Moreover, infected dams of IP, DGNP and TE groups had similarly high cerebral cyst loads $(842 \pm 480,950 \pm 320$ and $850 \pm 305$ cysts per brain, respectively), while DGNP/TE dams had significantly less cerebral cysts in brain tissue

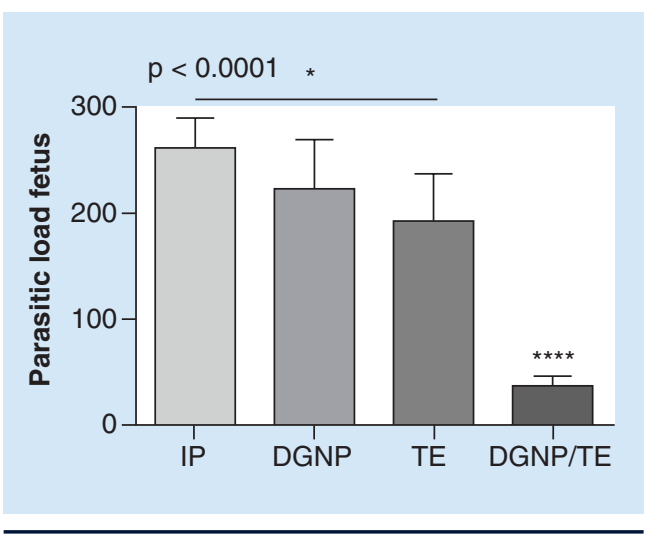

Figure 3. Parasite load in fetus. Parasite load was determined by real-time PCR in fetuses from nonimmunized mice (IP) or from mice immunized by the nasal route with nanoparticles (DGNP), total antigen extract (TE) or the vaccine formulation (DGNP/TE) and orally infected with 15 cysts of 76K T. gondii strain on day 11 of the gestation. Parasite load in fetus was evaluated at the 17th day of gestation (G17). Data are representative results of two independent experiments ( $n=6 /$ group) and are expressed as the mean of number of parasites multiplied by $10^{2} / 0.1 \mathrm{mg}$ of fetal DNA \pm SD (Kruskal-Wallis $\left.{ }^{*} \mathrm{p}<0.05 ;{ }^{* * * *} \mathrm{p}<0.0001\right)$. DGNP: Di-palmitoyl phosphatidyl glycerolloaded nanoparticles; IP: Infected pregnant; NIP: Noninfected pregnant; TE: Total antigen extract. 
(A)

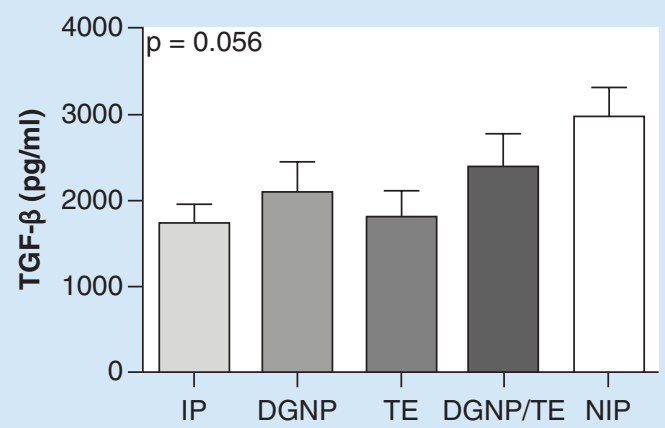

(C)

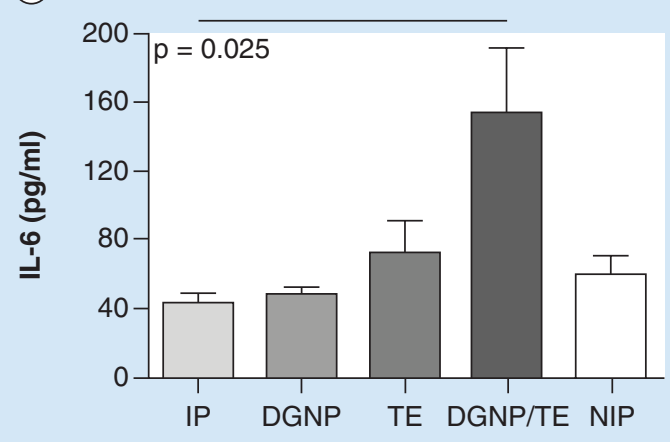

(B)

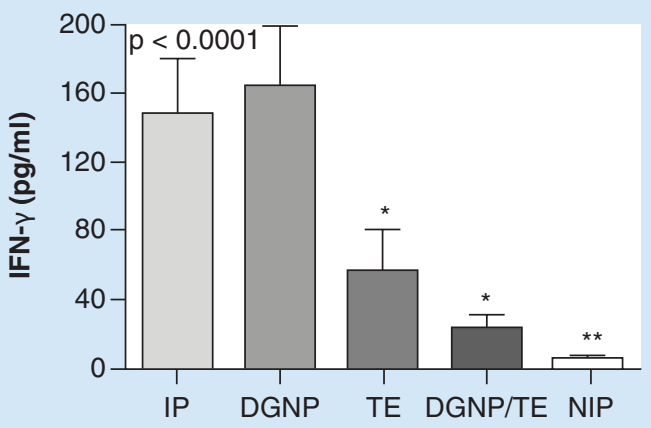

(D)

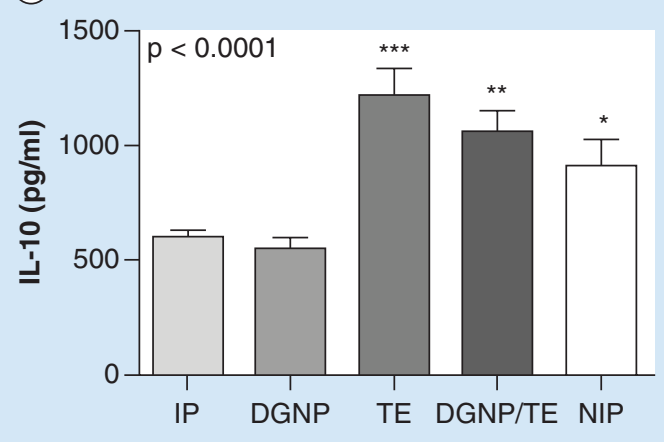

Figure 4. Placental cellular immune response. Placentas were collected on G17 from nonimmunized mice (IP), mice immunized by the nasal route with nanoparticles (DGNP), TE or the vaccine formulation (DGNP/TE) and orally infected with 15 cysts of 76K T. gondii strain on day 11 of the gestation, or nonimmunized, noninfected mice (NIP). TGF- $\beta$ (A), IFN- $\gamma$ (B), IL-6 (C) and IL-10 (D) cytokines were measured by ELISA from placental tissues. Data are representative results of two independent experiments $(n=5 / g r o u p)$ and are expressed as the mean/placenta \pm SD (Kruskal-Wallis ${ }^{*} \mathrm{p}<0.05 ;{ }^{* *} \mathrm{p}<0.01 ;{ }^{* * *} \mathrm{p}<0.001$ ).

DGNP: Di-palmitoyl phosphatidyl glycerol-loaded nanoparticle; IP: Infected pregnant; NIP: Noninfected pregnant; TE: Total antigen extract.

$(407 \pm 157)$, corresponding to a $55 \%$ decrease in cysts in comparison with dams immunized with DGNP or TE. This difference was significant with a p-value $=0.009$ (Kruskal-Wallis) (Figure 6B).

Results showed the potential of DGNP/ TE vaccine after intranasal delivery to reduce the development of tissue cysts in neonates born to vaccinated mice and in dams. Taken together, these results show that DGNP/TE is a powerful vaccine candidate against congenital toxoplasmosis.

\section{Discussion}

Development of vaccines to prevent congenital infections is a major public health priority. Toxoplasma gondii is best known as a cause of congenital disease, which can occur when a woman is primarily infected during pregnancy. While many vaccine candidates induce protective immunity against acute and/or chronic $T$. gondii infection in animals, a few of them were tested against congenital infection and/or proved their efficacy for the prevention of abortion. Indeed, a number of vaccine candidates including surface antigen (SAG), rhoptries (ROP), micronemes (MIC) and dense granules were evaluated over the years with respect to their induction of a protective immune response against chronic and/or congenital toxoplasmosis [23-29]. However, only partial protection was achieved in these studies, providing evidence of the efficacy of a multiple antigen vaccine for the induction of a protective immune response against congenital toxoplasmosis. In this sense few vaccine candidates formulated with the whole parasite or total proteins were tested with promising results against chronic infection but none has been tested and/ or proved efficacy against congenital infection [30-32]. This supports the importance of 
(A)

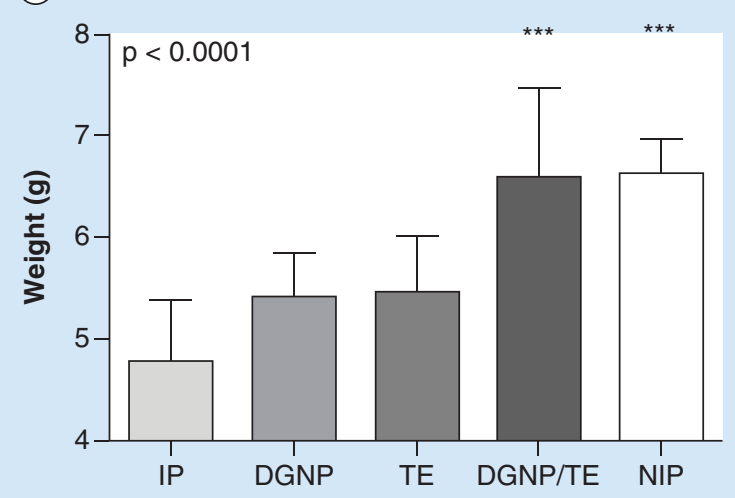

(C)
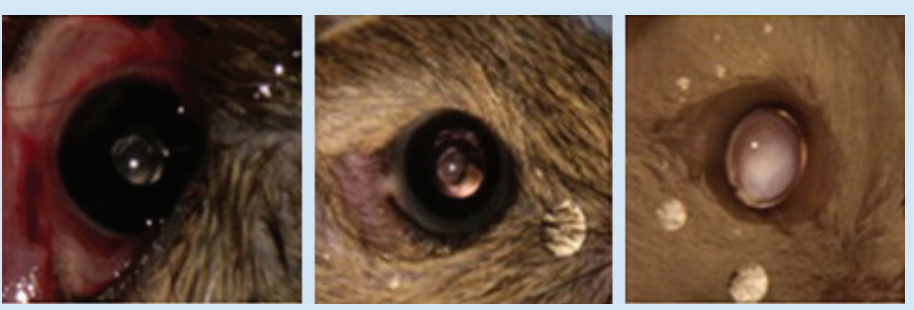

(D)

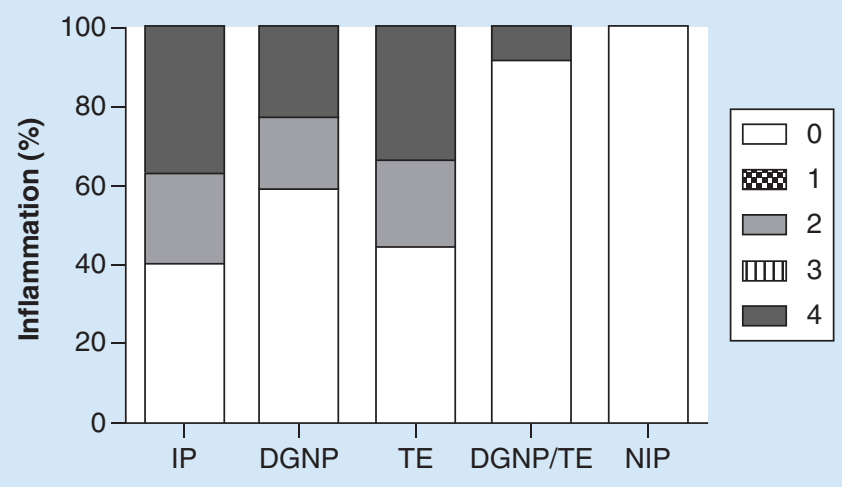

Figure 5. Growth and ocular inflammation in offspring. Analysis of the weight of 2-week-old offspring from nonimmunized mice (IP), mice immunized by the nasal route with nanoparticles (DGNP), TE or the vaccine formulation (DGNP/TE) and orally infected with 15 cysts of 76K T. gondii strain on day 11 of the gestation, or nonimmunized, noninfected mice (NIP). Data are representative results of two independent experiments and are expressed as the mean \pm SD (Kruskal-Wallis $p<0.0001 ; * * * p<0.001$ ) (A). Image of 8-week-old offspring from infected mice nasally vaccinated with DGNP/TE (left) or with DGNP (right) (B).

(C \& D) Ophthalmologic clinical signs in offspring at 8 weeks of age from nonimmunized mice (IP), mice immunized by the nasal route with nanoparticles (DGNP), total antigen extract (TE) or the vaccine formulation (DGNP/TE) and orally infected with 15 cysts of 76K T. gondii strain on day 11 of the gestation, or nonimmunized, noninfected mice (NIP). The determination of ophthalmologic clinical signs was performed under loupe on each eye of each mouse. Nuclear cataract (opacification of the lens nucleus) in eye of offspring from DGNP-immunized mouse (left) and from TE-immunized mouse (middle); normal lens in eye of offspring from DGNP/TE-immunized mouse (right) (C). Proportion of ocular inflammation scores: 0 : noninflammatory signal; 1: Tyndall in the anterior chamber or modest vitreous; 2: Tyndall in the anterior chamber or severe vitreous and/or dilatation of iris vessels and/or conjunctiva-sclera (cataract in the posterior capsule); 3: cornea troubles and precipitate retrocornea and/or hyalite very severe; 4 : secondary cataract (D). DGNP: Di-palmitoyl phosphatidyl glycerol-loaded nanoparticle; IP: Infected pregnant; NIP: Noninfected pregnant; TE: Total antigen extract. 
adjuvants and carriers for enhancing induction of protective immunity.

Immunity of pregnant mice highly differs from nonpregnant animals. However, the immune response induced by primary Toxoplasma infection confers lifelong protection against congenital toxoplasmosis suggesting that it is possible to develop a safe and effective vaccine.

In an attempt to protect both fetus and mother from congenital infection, pertinent vaccination strategies have to be explored to find the most immunogenic Toxoplasma gondii antigen(s), optimal carriers and adjuvants, as well as the best immunization route.

Over the past decade nanospheres have received attention as potential delivery vehicles for vaccine antigens that can also act as adjuvants and modulate the immune response to the antigens. Their properties also make them suitable for the delivery of antigens at mucosal surfaces. Recently, the use of porous nanoparticles as nasal delivery systems for antigens has been shown to protect mice from acute and chronic infection against $T$. gondii $[16]$.

These encouraging results led us to test their efficacy in congenital toxoplasmosis. It is important to have a model that resembles the human infection as closely as possible regarding vertical transmission and fetal damage. If many animal models (rhesus monkey, rate, sheep...) have been used to test efficacy of vaccine against $T$. gondii, mouse is the most frequently studied experimental model of congenital toxoplasmosis. Histology of human and rodent placentas is very similar and the mouse is the most frequently studied experimental model of congenital toxoplasmosis [33]. Roberts and Alexander demonstrated that mice chronically infected before pregnancy developed immunity capable of totally protecting their embryos after reinfection during pregnancy and concluded that mouse can be used as a model of human or ovine congenital T. gondii infection suitable for testing putative vaccines [34].

Administered intranasally DGNP/TE is a potent, effective and successful vaccine against congenital toxoplasmosis in mice. During gestation, on day G17, fetuses from DGNP/ TE-vaccinated mice were significantly less infected than other infected groups with a reduction of parasitemia of up to $86 \%$. All offspring born to vaccinated dams had normal weight growth, presented a significantly lower ocular pathological score, and significantly fewer cysts in their brains than offspring born to other groups of mice. This protection was associated with production of IL- 6 and a reduced secretion of IFN- $\gamma$ by placental tissue, suggesting that a protective Th1 immune
(A)

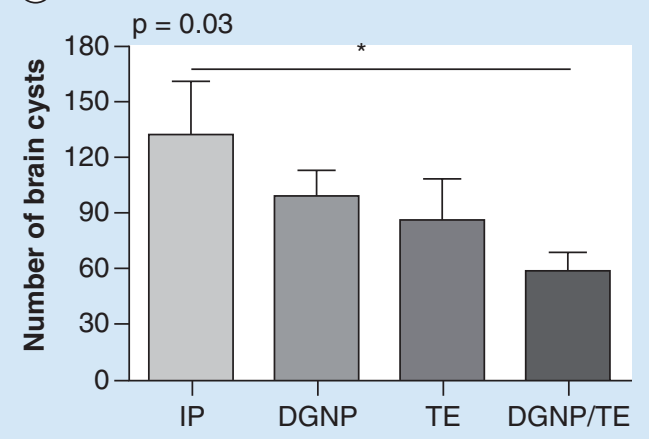

(B)

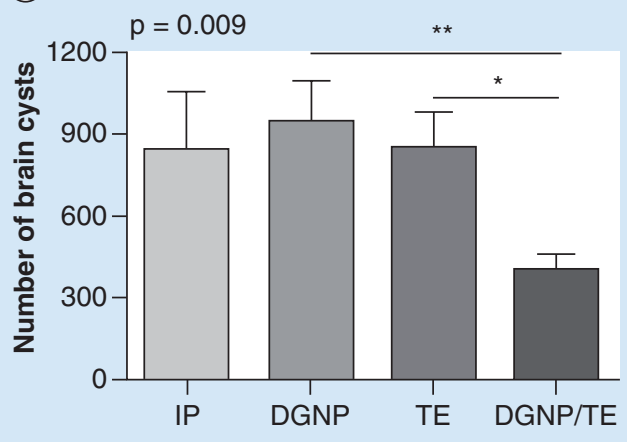

Figure 6. Cyst load in brain of offspring and dams. Mice were nonimmunized mice (IP) or immunized by the nasal route with nanoparticles only (DGNP), Toxoplasma proteins extract only (TE) or the nanoparticle vaccine formulation (DGNP/TE) and then orally infected with 15 cysts of 76K T. gondii strain on day 11 of the gestation. Cysts were counted in brain tissue of offspring 2 months after birth (A) and of dams 2 months after infection (B). Data are representative results of two independent experiments and are expressed as the mean \pm SD (Kruskal-Wallis, respectively, $\mathrm{p}=0.03$ and $\mathrm{p}=0.009 ;{ }^{*} \mathrm{p}<0.05 ;{ }^{* *} \mathrm{p}<0.01$ ). DGNP: Di-palmitoyl phosphatidyl glycerol-loaded nanoparticle; IP: Infected pregnant; NIP: Noninfected pregnant; TE: Total antigen extract. 
response induced by vaccination observed in a previous study is equilibrated during pregnancy [35]. Indeed during initial and chronic infection, T. gondii induces a strongly polarized Th1 response that is highly effective in host protection. During pregnancy, an early Th1type inflammatory environment is also essential for successful implantation and control of infectious diseases. However, this Th1 immune response may be a cause of immune-mediated failure of pregnancy, so a delicate balance of the Th1/Th2 T-cell cytokines is necessary to simultaneously maintain pregnancy and resistance to the parasite. This suggests that abortion may be induced directly by $T$. gondii placental infection, but also indirectly due to a strong Th1 or low Th2 maternal immune response [36-39].

The success of our vaccine is probably due to the encapsulation of all Toxoplasma antigens in the same nanoparticle-based vaccine and the delivery of these antigens in airway mucosa [16].

Only a few studies have reported the use of a sustained-release vaccine formulation against toxoplasmosis. Chuang et al. observed a long-lasting protective immunity against $T$. gondii in nonpregnant BALB/c mice after Poly(D,L-Lactideco-glycolide) (PLG) microparticle encapsulation of the SAG1 and SAG2 protein antigens [40]. However, Stanley et al. showed no protection in pregnant sheep given an intranasal PLG microparticle vaccine containing a tachyzoite extract plus cholera toxin [41]. To our knowledge, our study is the first in which Toxoplasma antigens associated with nanoparticles have been shown to protect against congenital toxoplasmosis.

Furthermore, nasal administration of DGNP/ TE showed that this route of administration of antigens protects against an oral challenge with the parasite and offers a promising alternative, as intranasal delivery of vaccines presents some distinct advantages over parenteral or other routes of vaccine administration. This noninvasive route is effective in systemic and mucosal immune responses by interacting with the nasal associated lymphoid tissue (NALT) [42]. Several studies have shown that vaccination at local mucosal surfaces, particularly the nasal surface, is an effective regimen for the stimulation of mucosal and systemic immune responses and is a promising and rational approach for vaccine development against $T$. gondii [43-46].

Beforehand, we showed that these nanoparticles increase the delivery of antigens into epithelial cells, therefore inducing a strong protective immune response in $T$. gondii chronic infection [16]. The in vivo biodistribution measurements in this study demonstrated the presence of nanoparticles in the nose $6 \mathrm{~h}$ after administration. After $24 \mathrm{~h}$, nanoparticles were undetectable from tissues, but present in the feces suggesting rapid and total elimination of the nanoparticles. Antigens contained in nasal vaccines usually reside in the nose for a matter of minutes [47]. This residence time was increased to $6 \mathrm{~h}$ by the DGNP and could lead, together with the intracellular delivery capabilities of DGNP [16], to an improved immunogenicity.

These results associated with safety issues of the total elimination of the nanoparticles confirm the strong interest of such nanoparticles as vaccine adjuvant.

Further studies will be performed with the DGNP/TE formulation to determine the degradation and/or the processing of proteins by immune cells. The mechanisms implied in antigen delivery via the mucosa, and specifically the question of what cells are involved, will also be examined.

These results provide support for future studies of congenital vaccines and confirm that these biodegradable particles can be used as a versatile approach to design vaccine carriers against vertically transmitted infections.

\section{Conclusion \& future perspective}

This publication presents the ability of total Toxoplasma antigens encapsulated in maltodextrin/phospholipid nanoparticles and administered intranasally to protect against congenital toxoplasmosis in mice. These results are very encouraging - regarding the incidence of ovine and human congenital toxoplasmosis in the world and their consequences - and showed that nanoparticle vaccines hold great promise not only for congenital toxoplasmosis but also in the area of all congenital infectious diseases. In the upcoming months, this strategy will be applied to ovine congenital toxoplasmosis prior to first assay in humans.

Financial \& competing interests disclosure

This work was funded by the French ANR (Agence Nationale de la Recherche) through grant ANR-12EMMA-0032-02 entitled 'NanoToxo'. The Vietnamese Government and Hanoi Institute of Biotechnology for PhD financial support. The authors have no other relevant affiliations or financial involvement with any organization or 
entity with a financial interest in or financial conflict with the subject matter or materials discussed in the manuscript apart from those disclosed.

No writing assistance was utilized in the production of this manuscript.

Ethical conduct of research

The authors state that they have obtained appropriate institutional review board approval or have followed the principles outlined in the Declaration of Helsinki for all human or animal experimental investigations. In addition, for investigations involving human subjects, informed consent has been obtained from the participants involved.

\section{Open Access}

This work is licensed under the Creative Commons Attribution 4.0 License. To view a copy of this license, visit http://creativecommons.org/licenses/by/4.0/

\section{SUMMARY POINTS}

\section{Background}

- Congenital Toxoplasma gondii infection is a major public health problem, and vaccines are a major priority.

\section{Results}

- Nanoparticles were totally eliminated after nasal administration confirming the biocompatibility and the biodegradability of the vaccine.

- Fetuses from vaccinated mice were significantly protected in terms of parasitemia and clinical symptoms.

- A local modulation of the immune response was observed at placental level.

\section{Conclusion}

- Administered intranasally nanoparticle vaccine is a potent, effective and successful vaccine against congenital toxoplasmosis in mice.

\section{References}

Papers of special note have been highlighted as: •• of considerable interest

1 Griffiths KL, Khader SA. Novel vaccine approaches for protection against intracellular pathogens. Curr. Opin. Immunol. 28, 58-63 (2014).

2 WHO The World Health Report (2005).

3 Arvin AM, Fast P, Myers M, Plotkin S, Rabinovich R. Vaccine development to prevent cytomegalovirus disease: report from the National Vaccine Advisory Committee. Clin. Infect. dis. 39(2), 233-239 (2004).

4 Hotez PJ. Neglected infections of poverty in the United States of America. PLoS Negl. Trop. Dis. 2(6), e256 (2008).

5 Kieffer F, Wallon M. Congenital toxoplasmosis. Handb. Clin. Neurol. 112, 1099-1101 (2013).

6 Dunn D, Wallon M, Peyron F, Petersen E, Peckham C, Gilbert R. Mother-to-child transmission of toxoplasmosis: risk estimates for clinical counselling. Lancet 353(9167), 1829-1833 (1999).

7 Buxton D. Protozoan infections (Toxoplasma gondii, Neospora caninum and Sarcocystis spp.) in sheep and goats: recent advances. Vet. Res. 29(3-4), 289-310 (1998).
8 Blewett DA, Trees AJ. The epidemiology of ovine toxoplasmosis with especial respect to control. Br. Vet. J. 143(2), 128-135 (1987).

9 Wallon M, Peyron F, Cornu C et al. Congenital Toxoplasma infection: monthly prenatal screening decreases transmission rate and improves clinical outcome at age 3 years. CID 56(9), 1223-1231 (2013).

10 Prusa AR, Kasper DC, Pollak A, Olischar M, Gleiss A, Hayde M. Amniocentesis for the detection of congenital toxoplasmosis: results from the nationwide Austrian prenatal screening program. Clin. Microbiol. Infect. 21(2), 191e1-191e8 (2015).

11 Ismael AB, Dimier-Poisson I, Lebrun M, Dubremetz JF, Bout D, Mévélec MN. Mic1-3 Knockout Toxoplasma gondii is a successful vaccine against chronic and congenital toxoplasmosis in mice. J. Infect. Dis. 194(8), 1176-1183 (2006).

-• Demonstrates that Mic1-3KO, a mutant strain of Toxoplasma gondii $\mathrm{RH}$ that lacks the MIC1 and MIC3 genes, is a potent, effective and successful vaccine against acquired and congenital toxoplasmosis.

12 Mévélec MN, Ducournau C, Bassuny Ismael A et al. Mic1-3 Knockout Toxoplasma gondii is a good candidate for a vaccine against $T$. gondii-induced abortion in sheep. Vet. Res. 41(4), 49 (2010).
13 Joshi VB, Geary SM, Salem AK. Biodegradable particles as vaccine antigen delivery systems for stimulating cellular immune responses. Hum. Vacc. Immunother. 9, 2584-2590 (2013).

14 Sahdev P, Ochyl LJ, Moon JJ. Biomaterials for nanoparticle vaccine delivery systems. Pharmaceut. Res. 31, 2563-2582 (2014).

15 Cordeiro AS, Alonso MJ, de la Fuente M. Nanoengineering of vaccines using natural polysaccharides. Biotechnol. Adv. 33, 1279-1293 (2015).

16 Dimier-Poisson I, Carpentier R, Nguyen TTL, Dahmani F, Ducournau C, Betbeder D. Porous nanoparticles as delivery system of complex antigens for an effective vaccine against acute and chronic Toxoplasma gondii infection. Biomaterials 50, 164-175 (2015).

-• The first study to fully analyze the characterization of antigen loading into nanoparticles and to study their role as vectors in airway mucosa; furthermore, these formulations showed a high protection against toxoplasmosis infection in acute and chronic infection.

17 Paillard A, Passirani C, Saulnier P et al. Positively-charged, porous, polysaccharide nanoparticles loaded with anionic molecules behave as 'stealth' cationic nanocarriers. Pharm. Res. 27, 126-133 (2010). 
18 Ismael AB, Sekkai D, Collin C, Bout D, Mevelec MN. The MIC3 gene of Toxoplasma gondii is a novel potent vaccine candidate against toxoplasmosis. Infect. Immun. 71, 6222-6228 (2003).

19 Ajzenberg D, Cogne N, Paris L et al. Genotype of 86 Toxoplasma gondii isolates associated with human congenital toxoplasmosis, and correlation with clinical findings. J. Infect. Dis. 186, 684-689 (2002).

20 Kupferschmidt O, Krüger D, Held TK, Ellerbrok H, Siegert W, Janitschke K. Quantitative detection of Toxoplasma gondii DNA in human body fluids by TaqMan polymerase chain reaction. Clin. Microbiol. Infect. 7, 120-124 (2001).

21 Sauer A, Lahmar I, Scholler M et al. Development of murine models of ocular toxoplasmosis and preliminary results of ocular inflammatory transcriptome. J. Fr. Ophtalmol. 32, 742-749 (2009).

22 Shiono Y, Mun HS, He N et al. Maternalfetal transmission of Toxoplasma gondii in interferon-gamma deficient mice. Parasitol. Int. 56, 141-148 (2007).

23 Zheng B, Lu S, Tong Q, Kong Q, Lou D. The virulence-related rhoptry protein 5 (ROP5) of Toxoplasma gondii is a novel vaccine candidate against toxoplasmosis in mice. Vaccine 31(41), 4578-4584 (2013).

24 Yuan ZG, Ren D, Zhou DH et al. Evaluation of protective effect of pVAX-TgMIC13 plasmid against acute and chronic Toxoplasma gondii infection in a murine model. Vaccine 31(31), 3135-3139 (2013).

25 Döșkaya M, Kalantari-Dehaghi M, Walsh $\mathrm{CM}$ et al. GRA1 protein vaccine confers better immune response compared with codon-optimized GRA1 DNA vaccine. Vaccine 25, 1824-1837 (2007).

26 Yuan ZG, Zhang XX, He XH et al. Protective immunity induced by Toxoplasma gondii rhoptry protein 16 against toxoplasmosis in mice. Clin. Vaccine Immunol. 18, 119-124 (2011).

27 Yuan ZG, Zhang XX, Lin RQ et al. Protective effect against toxoplasmosis in mice induced by DNA immunization with gene encoding Toxoplasma gondii ROP18. Vaccine 29, 6614-6619 (2011).

28 Xu Y, Zhang NZ, Tan QD et al. Evaluation of immuno-efficacy of a novel DNA vaccine encoding Toxoplasma gondii rhoptry protein 38 (TgROP38) against chronic toxoplasmosis in a murine model. BMC Infect. Dis. 14, 525-532 (2014).

29 Gong P, Cao L, Guo Y et al. Toxoplasma gondii: Protective immunity induced by a DNA vaccine expressing GRA1 and MIC3 against toxoplasmosis in BALB/c mice. Exp. Parasitol. 166, 131-136 (2016).

30 Zhao Y, Huang B, Huang S et al. Evaluation of the adjuvant effect of pidotimod on the immune protection induced by UVattenuated Toxoplasma gondii in mouse models. Parasitol. Res. 112, 3151-3160 (2013).

31 Khorshidvand Z, Shahabi S, Mohamadzade H, Daryani A, Hazrati Tappeh K. Mixture of alum-naloxone and alum-naltrexone as a novel adjuvant elicits immune responses for Toxoplasma gondii lysate antigen in BALB/c mice. Exp. Parasitol. 162, 28-34 (2016).

32 El-Malky M, Shaohong L, Kumagai T et al. Protective effect of vaccination with Toxoplasma lysate antigen and $\mathrm{CpG}$ as an adjuvant against Toxoplasma gondii in susceptible C57BL/6 mice. Microbiol. Immunol. 49, 639-646 (2005).

33 Darcy F, Zenner L. Experimental models of toxoplasmosis. Res. Immunol. 144(1), 16-23 (1993).

34 Roberts CW, Alexander J. Studies on a murine model of congenital toxoplasmosis: vertical disease transmission only occur in $\mathrm{BALB} / \mathrm{c}$ mice for the first time during pregnancy. Parasitology 104, 19-23 (1992).

35 Chaouat G, Ledée-Bataille N, Dubanchet $S$, Zourbas S, Sandra O, Martal J. Th1/Th2 paradigm in pregnancy: paradigm lost? Cytokines in pregnancy/early abortion: reexamining the Th1/Th2 paradigm. Int. Arch. Allergy Immunol. 134, 93-119 (2004).

36 Denkers EY, Gazzinelli RT. Regulation and function of T-cell-mediated immunity during Toxoplasma gondii infection. Clin. Microbiol. Rev. 11, 569-588 (1998).

37 Dupont CD, Christian DA, Hunter CA. Immune response and immunopathology during toxoplasmosis. Semin. Immunopathol. 34, 793-813 (2012).

38 Egan CE, Cohen SB, Denkers EY. Insights into inflammatory bowel disease using Toxoplasma gondii as an infectious trigger. Immunol. Cell. Biol. 90, 668-675 (2012).

39 Senegas A, Villard O, Neuville A et al. Toxoplasma gondii-induced foetal resorption in mice involves interferon-gamma-induced apoptosis and spiral artery dilation at the maternofoetal interface. Int. J. Parasitol. 39, 481-487 (2009).

Chuang SC, Ko JC, Chen CP, Du JT, Yang $\mathrm{CD}$. Induction of long-lasting protective immunity against Toxoplasma gondii in $\mathrm{BALB} / \mathrm{c}$ mice by recombinant surface antigen 1 protein encapsulated in poly (lactide-coglycolide) microparticles. Parasit. Vectors 6 , 34-46 (2013).
41 Stanley AC, Buxton D, Innes EA, Huntley JF. Intranasal immunisation with Toxoplasma gondii tachyzoite antigen encapsulated into PLG microspheres induces humoral and cell-mediated immunity in sheep. Vaccine 22, 3929-3941 (2004).

-. Shows that intranasal immunization of an extract of toxoplasma tachyzoite proteins including SAG1 using a Poly(D,L-Lactideco-glycolide) (PLG) microparticulate delivery system targets directly nasal associated lymphoid tissue (NALT) and induces specific humoral and cellular immune responses.

42 Neutra MR, Kozlowski PA. Mucosal vaccines: the promise and the challenge. $N a t$. Rev. Immunol. 6, 148-158 (2006).

43 EL-Malky MA, Al-Harthi SA, Mohamed RT, EL Bali MA, Saudy NS. Vaccination with Toxoplasma lysate antigen and $\mathrm{CpG}$ oligodeoxynucleotides: comparison of immune responses in intranasal versus intramuscular administrations. Parasitol. Res. 113, 2277-2284 (2014).

-• Shows that intranasal administration of Toxoplasma lysate antigen with $\mathrm{CpG}$ oligodeoxynucleotides induces higher levels of Th1 humoral response and protection than those obtained after intramuscular administration.

44 Igarashi M, Zulpo DL, Cunha IA et al. Toxoplasma gondii: humoral and cellular immune response of BALB/c mice immunized via intranasal route with rTgROP2. Rev. Bras. Parasitol. Vet. 19, 210-216 (2010).

45 Wang H, He S, Yao Y et al. Toxoplasma gondii: protective effect of an intranasal SAG1 and MIC4 DNA vaccine in mice. Exp. Parasitol 122, 226-232 (2009).

46 Cong H, Yuan Q, Zhao Q et al. Comparative efficacy of a multi-epitope DNA vaccine via intranasal, peroral, and intramuscular delivery against lethal Toxoplasma gondii infection in mice. Parasit. Vectors 7, 145 (2014).

47 Bernocchi B, Carpentier R, Lantier I, Ducournau C, Dimier-Poisson I, Betbeder D. Mechanisms allowing protein delivery in nasal mucosa using NPL nanoparticles. J. Control Release 232, 42-50 (2016).

- Aims to better understand the interactions of NPL nanoparticles with the airway epithelial cells, their ability to cross this barrier and the dynamics of NPL and protein interaction with the nasal mucosa. 